\title{
Una strana colica renale in un paziente nefrolitiasico e ...non solo
}

\author{
T. Lusenti ${ }^{1}$, F. Fiorini ${ }^{2}$, A. D’Amelio ${ }^{3}$, A. Granata ${ }^{4}$, S. Andrulli ${ }^{5}$
}

${ }^{1}$ Nefrologia ed Ecografia Reni e Vie Urinarie, Centro Medico “Lazzaro Spallanzani”, Reggio Emilia

${ }^{2}$ U.O.C. Nefrologia e Dialisi, Rovigo

${ }^{3}$ U.O.C. Nefrologia e Dialisi, Lecce

${ }^{4}$ U.O.C. Nefrologia e Dialisi, Catania

${ }^{5}$ U.O.C. Nefrologia e Dialisi, Lecco

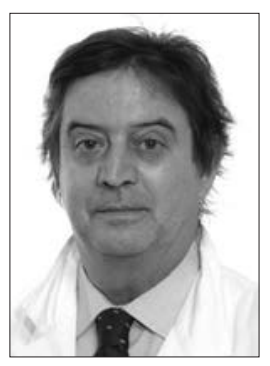

\section{Introduzione}

Descriviamo il caso di un paziente affetto da calcolosi urinaria bilaterale, con funzionalità renale nella norma, che si presenta al nostro ambulatorio ecografico per l'ennesimo episodio recidivo di colica renoureterale destra.

Tiziano Lusenti

\section{Caso clinico}

Il paziente maschio di 53 anni (che annoverava in anamnesi familiare una figlia diciannovenne affetta da nefrolitiasi), con anamnesi negativa per diabete e ipertensione, aveva presentato dall'età di 16 anni numerose coliche renoureterali da ambo i lati e per tale patologia era da allora sottoposto a follow-up clinico-ecografico presso l'ambulatorio nefrologico della calcolosi urinaria con diagnosi di calcolosi urica.

Nel 2003 aveva subito l'asportazione di un nevo all'avambraccio destro.

Nell'ottobre 2010 era giunto al Pronto Soccorso per dolore scapolare destro con riscontro radiologico di periartrite calcifica scapolo-omerale.

Per familiarità positiva da parte paterna per neoplasia intestinale aveva eseguito nel novembre 2010 screening colorettale che era risultato negativo.

Negli ultimi esami di laboratorio del dicembre 2010 presentava funzionalità renale nella norma (creatininemia $1,11 \mathrm{mg} / \mathrm{dL}, \mathrm{FG}$ calcolato per maschio non afroamericano secondo la formula dell'MDRD $70 \mathrm{ml} / \mathrm{m}^{\prime} / 1,73 \mathrm{mq}$, azotemia $34 \mathrm{mg} / \mathrm{dL}$ ),

Nell'esame chimico-fisico delle urine: pH 6,5, PS 1011, proteinuria ed $\mathrm{Hb}$ assenti, test ai nitriti ed esterasi leucocitaria negativi. Nella citofluorimetria urinaria: eritrociti 14 n./uL (v.n. 0-15), leucociti 6 n./uL (v.n. 0-20), batteriuria e cristalluria nei limiti.

Elettroliti serici, assetto lipidico, emocromo ed elettroforesi sieroproteica risultavano pure entro i range di normalità. L'uricemia era 5,6 mg/dL (vn. 3,4-8,5 mg/dL).

Nello screening metabolico per nefrolitiasi erano presenti: ipernatriuria ( $\mathrm{Na} 169 \mathrm{mmol} / 24 \mathrm{~h}$, v.n. fino a 100 $\mathrm{mmol} /$ die) e iperazoturia (9187 mg/24 h, v.n. $3000-8000$ $\mathrm{mg}$ /die), con calciuria, uricuria, ossaluria e citraturia nei limiti e volume urinario $>2 \mathrm{lt} / 24 \mathrm{~h}$. Era pertanto in terapia idropinica con indicazione a una dieta iposodica e con riduzione dell'apporto di proteine animali, associati a citrato di potassio.

Nel dicembre scorso si presenta presso il nostro ambulatorio nefrologico di ecografia lamentando l'insorgenza, 3 giorni prima, di colica renoureterale destra non accompagnata da febbre né ematuria.

Viene sottoposto ad esame ecografico dei reni e delle vie urinarie mediante ecografo Siemens Antares con sonda convex da 3,5 MHZ, che mostra reni in sede, di volume normale (asse longitudinale $>10 \mathrm{~m}$ da ambo i lati), con idronefrosi destra di II grado associata a lieve riduzione dello spessore parenchimale, in assenza di calcoli evidenziabili in ambito urinario (Fig. 1).

Il paziente viene indirizzato all'uro-TAC, che per maggior disponibilità temporale viene eseguita in sede extraospedaliera. Viene effettuata uro-TAC senza l'impiego del mdc che conferma la presenza di marcata idronefrosi a destra, senza apparente evidenza di calcoli in sede renale e ureterale.

Per il persistere della sintomatologia dolorosa e per una più precisa valutazione eziopatogenetica si ripresenta al 

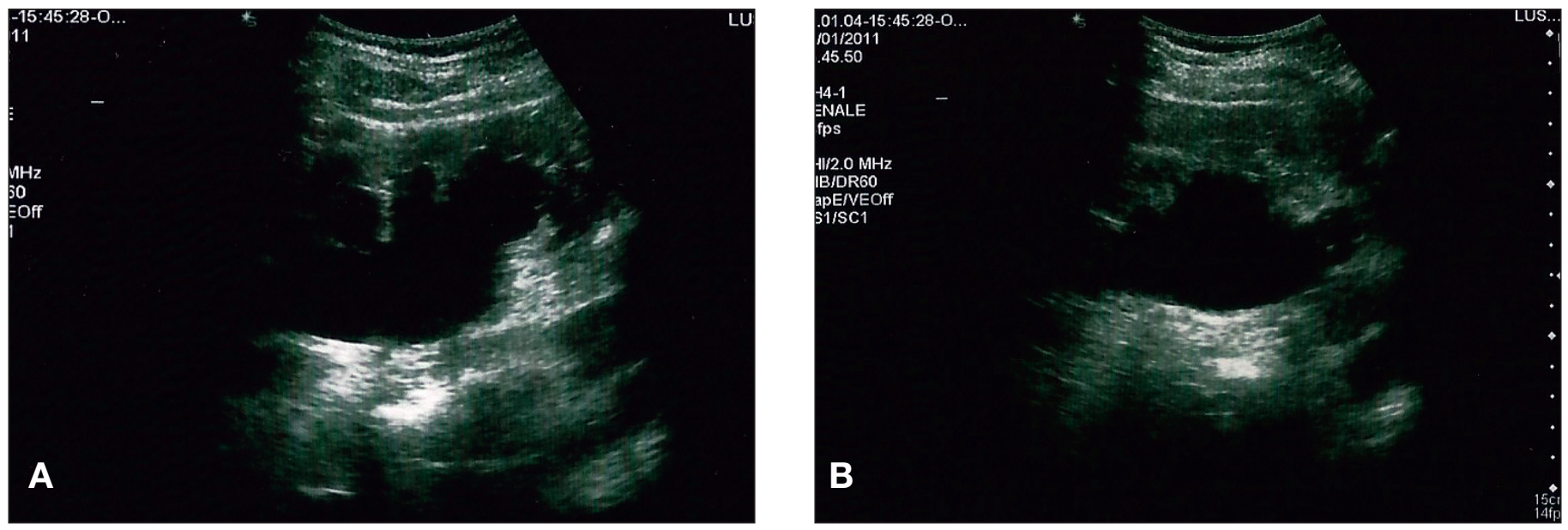

Fig. 1 - Ecografia renale. Sonda 3.5 MHZ. Le scansioni longitudinale A) e trasversa (B) mostrano idronefrosi di II grado del rene destro, con lieve riduzione del parenchima circostante, uretere non visualizzabile.
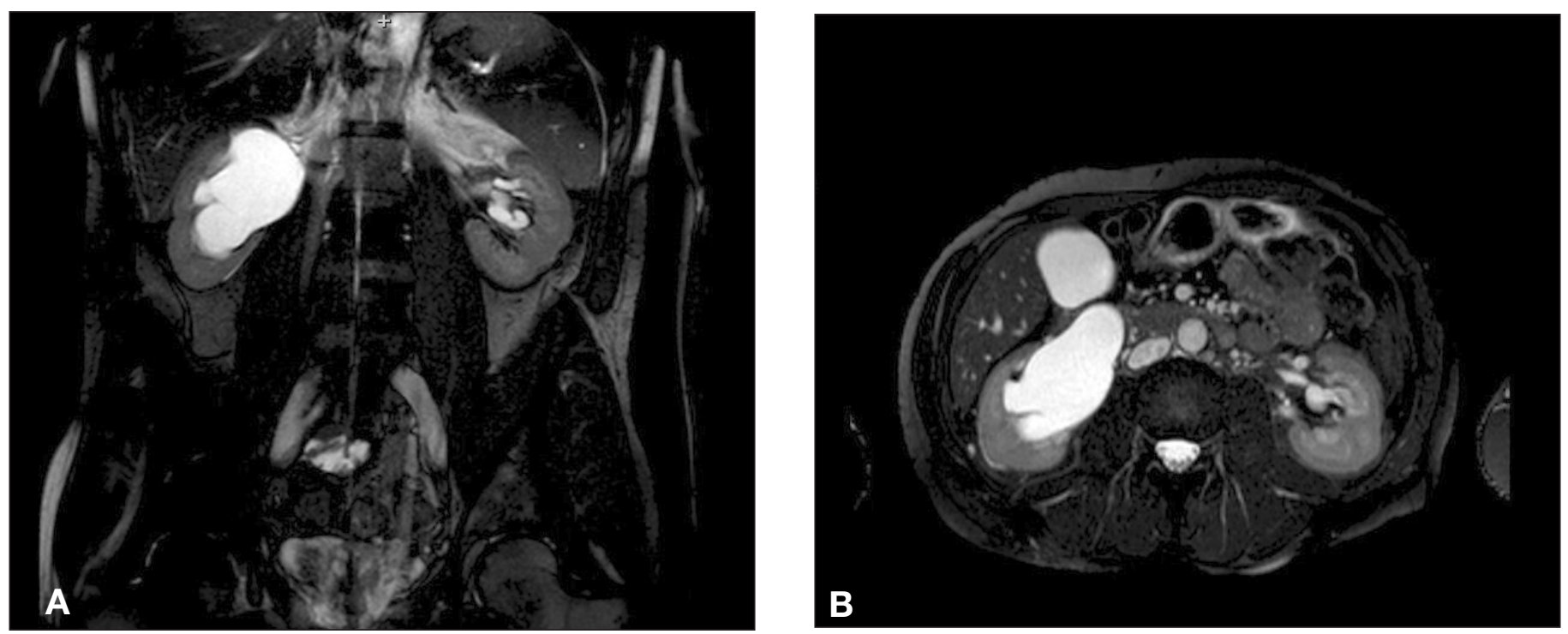

Fig. 2 - RMN addome superiore. Le scansioni corrispondenti $(A, B)$ evidenziano dilatazione delle cavità calicopieliche del rene destro fino a livello del giunto pieloureterale. Non difetti di riempimento imputabili a calcoli nelle vie escretrici dilatate. Uretere destro non ectasico in tutto il suo decorso.

nostro ambulatorio a visita di controllo. Indagato anamnesticamente riferisce un non meglio precisato episodio di allergia in corso di esame radiologico effettuato con l'uso del mezzo di contrasto iodato. In considerazione di ciò, e su indicazione del collega radiologo, viene inviato all'esecuzione di uro-RMN dell'addome.

L'indagine conferma il reperto dell'ecografia e della TAC addome di dilatazione delle cavità calico-pieliche del rene destro fino al giunto pieloureterale, non si rilevano difetti di riempimento imputabili a calcoli nelle vie escretrici dilatate, con uretere destro che in tutto il suo decorso non si mostra ectasico. Il quadro depone per ostruzione del giunto pieloureterale (OGPU) destro. $\mathrm{Si}$ conferma in aggiunta la lieve riduzione dello spessore parenchimale a livello del rene destro, già riscontrata nelle precedenti indagini (Fig. 2).

A conferma della diagnosi e per meglio valutare il grado di compromissione funzionale del rene interessato il paziente viene sottoposto a scintigrafia renale dinamica con stimolo diuretico con furosemide.

L'esame effettuato con 99mTc-MAG3 conferma la ridotta funzionalità renale destra con segni riferibili a dilatazione e ostruzione delle vie escretrici omolaterali significative per OGPU, con parametri morfo-funzionali del 

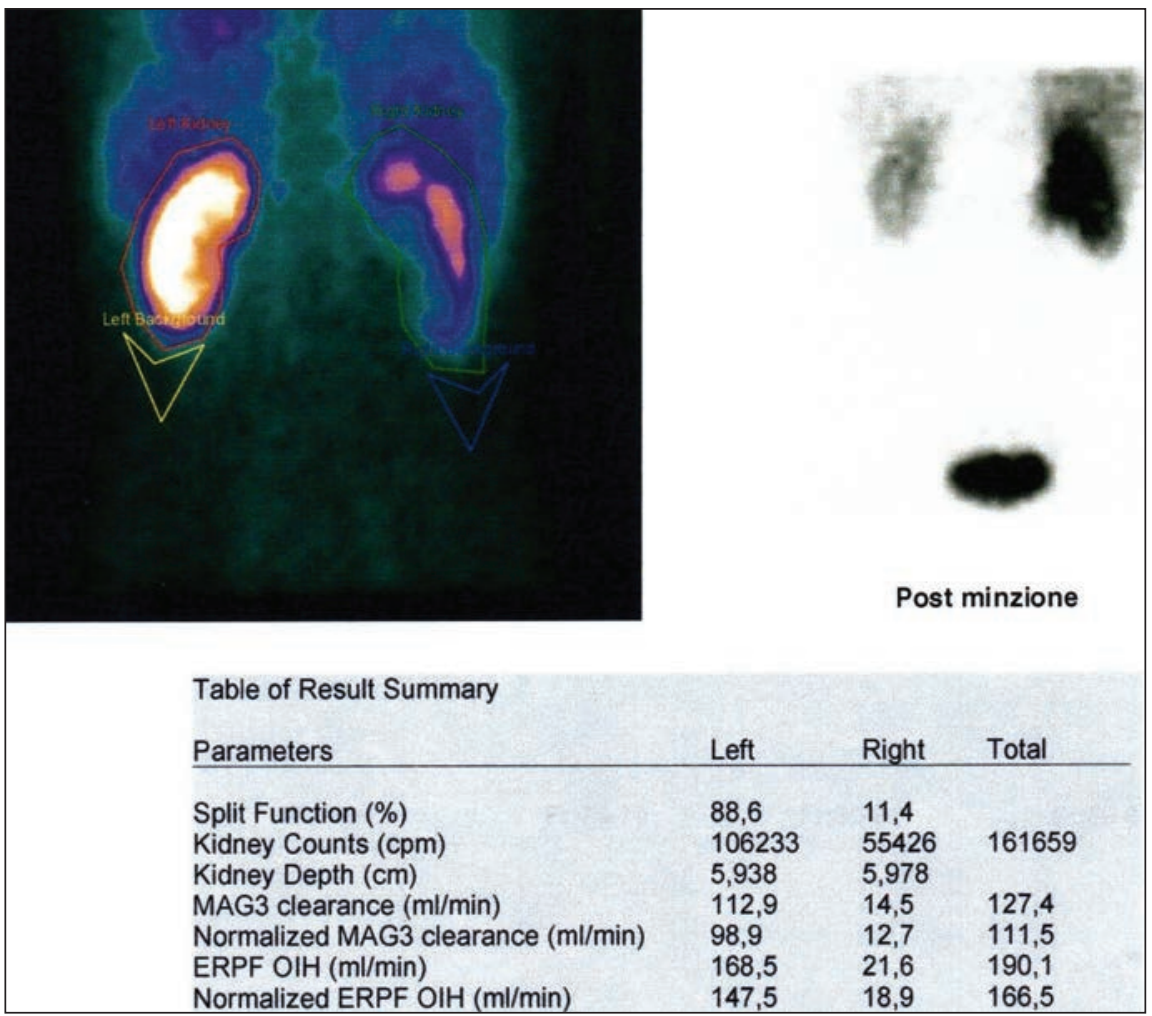

Fig. 3 - Scintigrafia renale dinamica che conferma la dilatazione delle vie escretrici a livello del rene destro con riduzione consensuale della funzionalità renale omolaterale (contributo funzionale percentuale: rene destro 11 , rene sinistro 89; clearance normalizzata MAG3 rene destro 13, rene sinistro $\left.99 \mathrm{ml} / \mathrm{m}^{\prime}\right)$. rene sinistro sostanzialmente nella norma (Fig. 3). Il paziente viene successivamente sottoposto ad intervento di pieloplastica del rene destro con buon esito. Nei mesi seguenti non ha, a tutt'oggi, presentato episodi recidivi di colica renale.

\section{Discussione}

L'uropatia ostruttiva (UO) riconosce classicamente in senso eziopatogenetico cause congenite e cause acquisite. Le UO da cause acquisite sono da difetti intrinseci o estrinseci alle vie escretrici. Tra le cause estrinseche vi sono le neoplasie (dell'utero e dell'ovaio nella femmina e vescicali e prostatiche nel maschio), le forme post-chirurgiche e la fibrosi retroperitoneale. I principali difetti intrinseci acquisiti sono l'ipertrofia prostatica benigna (IPB), gli uroteliomi e la calcolosi urinaria, come nel paziente qui riportato.

In riferimento al caso in esame si possono fare alcune considerazioni sui possibili "tranelli" nella diagnosi differenziale della causa dell'UO.

Occorre considerare che anamnesticamente i precedenti ripetuti episodi di colica renale si erano sempre risolti spontaneamente e in breve tempo con l'emissione di renella.

Negli esami ecografici effettuati dal paziente e segnata- mente anche in quello effettuato prima dell'ultima colica era segnalata una microlitiasi renale bilaterale per cui, poteva essere ipotizzabile, in prima istanza, la discesa in sede ureterale di uno o più microuroliti già individuati nelle vie escretrici del rene destro.

$\mathrm{E}$ infine, da ultimo ma non meno importante, in nessuna delle ecografie precedenti a cui il paziente si era sottoposto era mai stata segnalata una dilatazione calico-pielica di alcun grado, che potesse in qualche modo far sospettare un'altra causa di UO al di fuori della litiasi.

Le UO su base congenita si distinguono in cause anatomiche e funzionali. Le principali patologie su base funzionale sono il reflusso-vescico-ureterale e la vescica neurogena.

Le UO congenite su base anatomica sono: ostruzione del giunto pieloureterale (OGPU), megauretere e valvole dell'uretra (più spesso posteriore). L'OGPU rappresenta la causa più frequente di UO su base congenita. Pur essendo diagnosticata frequentemente già in epoca prenatale può manifestarsi più tardivamente anche in età adulta.

L'OGPU viene definita la patologia ostruttiva del giunto pieloureterale che determina un ostacolo al deflusso urinario a livello della giunzione pieloureterale dovuto a cause intrinseche (mal strutturazioni parietali per anomalie delle miocellule della tonaca muscolare, valvole, 
stenosi intrinseche, impianto alto dell'uretere, meccanismi neurogeni) o estrinseche (vasi anomali che incrociano il giunto pieloureterale, briglie, membrane avventiziali) (1).

$\mathrm{Si}$ riscontra con maggior frequenza nel sesso maschile (rapporto $\mathrm{M} / \mathrm{F}$ : $2 / 1$ ). In più della metà dei pazienti interessa il rene destro, fino al $10 \%$ dei casi può essere bilaterale $o$ associarsi a reflusso vescico-ureterale.

I sintomi possono essere già nel periodo neonatale una massa palpabile in addome $o$ in età più avanzata infezioni delle vie urinarie o calcolosi per l'associata stasi urinaria o dolori addominali che possono, come in questo caso, essere francamente di tipo colico.

La diagnosi mediante imaging si fonda in prima battuta sull'esame ecografico, e negli step successivi sulla scintigrafia renale e uro-TAC o uro-RMN.

L'ecografia evidenzia in questi casi un calico-pielectasia di grado più o meno marcato, con uretere non dilatato (assenza di idrouretere), spesso associata a riduzione dello spessore corticale del rene interessato (2). Nei casi dubbi un IR $>0,7$ può aiutare a differenziare le calico-pielectasie da cause ostruttive da quelle da cause non ostruttive (reflusso vescico-ureterale, vescica neurogena) (3).

In questi pazienti riveste particolare importanza per la diagnosi la scintigrafia renale dinamica con 99mTc-MAG3, che consente di misurare la compromissione funzionale del rene interessato e al tempo stesso di rilevare il rallentamento dei tempi di escrezione dell'isotopo, con prolungamento della fase escretoria anche dopo stimolo diuretico. Più recentemente TAC e RMN hanno incrementato la sensibilità diagnostica dell'imaging nel differenziare le forme ostruttive da quelle non ostruttive (4).

Il precoce risconoscimento di un'UO su base congenita risulta di particolare importanza anche per il nefrologo in considerazione delle possibili conseguenze in età adulta di tali uropatie malformative. A tal proposito, tra i numerosi studi effettuati, basta ricordare i risultati del lavoro di Zang e Bailey che in una casistica sufficientemente numerosa di 294 pazienti con nefropatia da reflusso, seguiti in un follow-up di 17 anni, hanno riscontrato un'incidenza di ipertensione del 18\% e una riduzione della funzione renale nel $24 \%$ dei pazienti esaminati (5).

In questi casi l'unica terapia efficace per la prevenzione del danno renale legato all'ostruzione resta ovviamente quella chirurgica e si basa sull'intervento di pieloplastica a livello del rene interessato o di incisione per via endoscopica del tratto stenotico (con maggior frequenza di recidive), che presentano però un outcome non sempre positivo, nelle diverse casistiche, riguardo alla preservazione della funzionalità del renale (6-8) .

In conclusione il caso qui riportato ribadisce come nei casi di UO l'ecografia renale costituisca il primo essenziale step diagnostico per inviare il paziente alle successive indagini di imaging (TAC, RMN, scintigrafia renale). Sottolinea inoltre il fatto che, in tema di diagnosi differenziale, nei pazienti con idronefrosi accanto alle più frequenti cause acquisite di UO quali calcolosi urinaria (8), IPB e neoplasie occorra sempre considerare le possibili cause congenite e tra queste in particolare l'OGPU.

\section{Riassunto}

Maschio di 56 anni con familiarità positiva per nefrolitiasi ed anamnesi positiva per numerosi episodi di colica renoureterale da ambo i lati, si presenta al nostro ambulatorio nefrologico di ecografia per ennesimo episodio recidivo di colica renale destra. All'ultimo controllo ecografico dei reni presentava microlitiasi bilaterale in assenza di dilatazione delle vie escretrici. $\mathrm{E}$ in terapia dietetico-idropinica associata a citrato di potassio. All'ecografia renale presenta idronefrosi del rene destro in assenza di idrouretere. La TAC, eseguita senza mezzo di contrasto per pregresso episodio di allergia al mdc in corso di precedente esame radiologico conferma la dilatazione delle vie escretrici a carico del rene destro. La successiva RMN dell'addome ribadisce l'idronefrosi destra riscontrata all'ecografia e alla TAC, sino alla giunzione pieloureterale con uretere a valle non dilatato che depongono per ostruzione del giunto pieloureterale (OGPU). Il paziente operato di plastica del giunto pieloureterale è al momento libero da coliche. Il caso riportato ribadisce come nei casi di idronefrosi accanto alle più comuni cause di uropatia ostruttiva (calcolosi, IPB, neoplasie) occorra sempre considerare le patologie congenite che possono manifestarsi anche in età adulta $\mathrm{e}$ tra queste in particolare la sindrome del giunto pieloureterale. In questi pazienti l'ecografia renale rappresenta un importante esame di primo livello in base alla quale indirizzare il paziente ai successivi step diagnostici di imaging e ai conseguenti provvedimenti terapeutici.

Parole chiave: Ostruzione giunto pieloureterale, Colica renale, Idronefrosi, Ecografia renale

\section{Indirizzo degli Autori:}

Tiziano Lusenti, MD

Nefrologia ed Ecografia Reni e Vie Urinarie

Centro Medico "Lazzaro Spallanzani”

Via F.lli Cervi 75/B

42124 Reggio Emilia

emauele.lusenti4@hotmail.it 


\section{TEST DI VERIFICA - 3}

1) Quale reperto ecografico deve far sospettare l'OGPU in caso di idronefrosi:

a) Assenza di calicectasia

b) Assenza di idrouretere

c) Idronefrosi bilaterale

d) Rene non visualizzabile

2) In considerazione della pregressa allergia al mezzo di contrasto iodato a quale altra indagine strumentale sull'apparato urinario avreste sottoposto il paziente:
a) Urografia
b) Angiografia renale selettiva
c) uro-RMN
d) Stratigrafia renale

3) Per la valutazione quantitativa della funzionalità separata dei due reni e in particolare del rene destro avreste richiesto:
a) Scintigrafia renale con $99 \mathrm{mTc}$-DMSA
b) Scintigrafia renale con 99mTC-MAG3
c) Urografia
d) Ecodoppler renale

Le risposte corrette alle domande sono a pag. 17

\section{Bibliografia}

1. Alberti C, Tizzani A. Patologia ostruttiva del giunto pieloureterale: alcune nuove acquisizioni sulla eziopatogenesi e sulla diagnostica. Urologia 2003; 70; 1-4: 5-16.

2. Shokeir AA. The diagnosis of upper urinary tract obstruction. BJU 1999; 83: 893-901.

3. Vade A, DubiaK C, McCarthy, et al. Resistivity index in the evaluation of infants with obstructive and non-obstructive pyelocalicectasia. J Ultrasound Med 1999; 18: 357-61.

4. Sfakianakis GN, Sfakianakis E. Renal scintigraphy in infants and in children. Urology 2001; 57: 1167-70.

5. Zielonko J, Studniarek M. Markuzewski M. MR urography of obstructive uropathy: diagnosis value of the method in selected clinical groups. Eur Radiol 2003; 13: 802-9.

6. Zang Y, Bailey RR. Long-term follow-up of adults with reflux nephropathy. NZ Med J 1995; 108: 142-44.

7. McAleer IM, Kaplan GW. Renal function before and after pyeloplasty: does it improve? J Urol 1999; 162: 1041-44.

8. Chertin B, Rolle U, Farkas A, et al. Does early detection of ureteropelvic junction obstruction improve surgical outcome in terms of renal function? J Urol 1999; 162: 1037-40.

9. Lusenti T, Fiorini F, Barozzi L. Obstructive uropathy and acute renal failure due to ureteral calculus in renal graft: a case report. J Ultrasound 2009; 12: 128-32. 